\title{
On a Generalized Retarded Integral Inequality with Two Variables
}

\author{
Wu-Sheng Wang ${ }^{1,2}$ and Cai-Xia Shen ${ }^{1}$ \\ ${ }^{1}$ Department of Mathematics, Hechi College, Guangxi, Yizhou 546300, China \\ ${ }^{2}$ Department of Mathematics, Sichuan University, Chengdu, Sichuan 610064, China
}

Correspondence should be addressed to Wu-Sheng Wang,wang4896@126.com

Received 16 November 2007; Accepted 22 April 2008

Recommended by Wing-Sum Cheung

This paper improves Pachpatte's results on linear integral inequalities with two variables, and gives an estimation for a general form of nonlinear integral inequality with two variables. This paper does not require monotonicity of known functions. The result of this paper can be applied to discuss on boundedness and uniqueness for a integrodifferential equation.

Copyright (C) 2008 W.-S. Wang and C.-X. Shen. This is an open access article distributed under the Creative Commons Attribution License, which permits unrestricted use, distribution, and reproduction in any medium, provided the original work is properly cited.

\section{Introduction}

Gronwall-Bellman inequality [1,2] is an important tool in the study of existence, uniqueness, boundedness, stability, and other qualitative properties of solutions of differential equations and integral equations. There can be found a lot of its generalizations in various cases from literature (see, e.g., [1-12]). In [11], Pachpatte obtained an estimation for the integral inequality

$$
u(x, y) \leq a(x, y)+\int_{0}^{x} \int_{0}^{y} f(s, t)\left[u(s, t)+\int_{0}^{s} \int_{0}^{t} g(s, t, \sigma, \tau) u(\sigma, \tau) d \tau d \sigma\right] d t d s
$$

His results were applied to a partial integrodifferential equation:

$$
\begin{gathered}
u_{x y}(x, y)=F\left(x, y, u(x, y), \int_{0}^{x} \int_{0}^{y} h(x, y, \tau, \sigma, u(x, y)) d \tau d \sigma\right), \\
u\left(x, y_{0}\right)=\alpha(x), \quad u\left(x_{0}, y\right)=\beta(y),
\end{gathered}
$$

for boundedness and uniqueness of solutions. 
In this paper, we discuss a more general form of integral inequality:

$$
\begin{aligned}
& \psi(u(x, y)) \\
& \quad \leq a(x, y)+\int_{b\left(x_{0}\right)}^{b(x)} \int_{c\left(y_{0}\right)}^{c(y)} f(x, y, s, t)\left[\varphi_{1}(u(s, t))+\int_{b\left(x_{0}\right)}^{s} \int_{c\left(y_{0}\right)}^{t} g(s, t, \sigma, \tau) \varphi_{2}(u(\sigma, \tau)) d \tau d \sigma\right] d t d s
\end{aligned}
$$

for all $(x, y) \in\left[x_{0}, x_{1}\right) \times\left[y_{0}, y_{1}\right)$. Obviously, $u$ appears linearly in (1.1), but in our (1.3) it is generalized to nonlinear terms: $\varphi_{1}(u(s, t))$ and $\varphi_{2}(u(s, t))$. Our strategy is to monotonize functions $\varphi_{i}$ s with other two nondecreasing ones such that one has stronger monotonicity than the other. We apply our estimation to an integrodifferential equation, which looks similar to (1.2) but includes delays, and give boundedness and uniqueness of solutions.

\section{Main result}

Throughout this paper, $x_{0}, x_{1}, y_{0}, y_{1} \in \mathbf{R}$ are given numbers. Let $\mathbf{R}_{+}:=[0, \infty), I:=\left[x_{0}, x_{1}\right), J:=$ $\left[y_{0}, y_{1}\right)$, and $\Lambda:=I \times J \subset \mathbf{R}^{2}$. Consider inequality (1.3), where we suppose that $\psi \in C^{0}\left(\mathbf{R}_{+}, \mathbf{R}_{+}\right)$ is strictly increasing such that $\psi(\infty)=\infty, b \in C^{1}(I, I)$, and $c \in C^{1}(J, J)$ are nondecreasing, such that $b(x) \leq x$ and $c(y) \leq y, a \in C^{1}\left(\Lambda, \mathbf{R}_{+}\right), f \in C^{0}\left(\Lambda^{2}, \mathbf{R}_{+}\right)$, and $g(x, y, s, t) \in C^{0}\left(\Lambda^{2}, \mathbf{R}_{+}\right)$are given, and $\varphi_{i} \in C^{0}\left(\mathbf{R}_{+}, \mathbf{R}_{+}\right)(i=1,2)$ are functions satisfying $\varphi_{i}(0)=0$ and $\varphi_{i}(u)>0$ for all $u>0$.

Define functions

$$
\begin{aligned}
w_{1}(s) & :=\max _{\tau \in[0, s]}\left\{\varphi_{1}(\tau)\right\}, \\
w_{2}(s) & :=\max _{\tau \in[0, s]}\left\{\varphi_{2}(\tau) / w_{1}(\tau)\right\} w_{1}(s), \\
\phi(s) & :=w_{2}(s) / w_{1}(s) .
\end{aligned}
$$

Obviously, $w_{1}, w_{2}$, and $\phi$ in (2.1) are all nondecreasing and nonnegative functions and satisfy $w_{i}(s) \geq \varphi_{i}(s), i=1,2$. Let

$$
\begin{aligned}
W_{1}(u) & =\int_{1}^{u} \frac{d s}{w_{1}\left(\psi^{-1}(s)\right)}, \\
W_{2}(u) & =\int_{1}^{u} \frac{d s}{w_{2}\left(\psi^{-1}(s)\right)}, \\
\Phi(u) & =\int_{W_{1}(1)}^{u} \frac{d s}{\phi\left(\psi^{-1}\left(W_{1}^{-1}(s)\right)\right)} .
\end{aligned}
$$

Obviously, $W_{1}, W_{2}$, and $\Phi$ are strictly increasing in $u>0$, and therefore the inverses $W_{1}^{-1}, W_{2}^{-1}$, and $\Phi^{-1}$ are well defined, continuous, and increasing. We note that

$$
\begin{aligned}
\Phi(u) & =\int_{W_{1}(1)}^{u} \frac{d x}{\phi\left(\psi^{-1}\left(W_{1}^{-1}(x)\right)\right)} \\
& =\int_{W_{1}(1)}^{u} \frac{w_{1}\left(\psi^{-1}\left(W_{1}^{-1}(x)\right)\right) d x}{w_{2}\left(\psi^{-1}\left(W_{1}^{-1}(x)\right)\right)} \\
& =\int_{1}^{W_{1}^{-1}(u)} \frac{d x}{w_{2}\left(\psi^{-1}(x)\right)}=W_{2}\left(W_{1}^{-1}(u)\right) .
\end{aligned}
$$


Furthermore, let $\tilde{f}(x, y, s, t):=\max _{\tau \in\left[x_{0}, x\right]} f(\tau, y, s, t)$, which is also nondecreasing in $x$ for each fixed $y, s$, and $t$ and satisfies $\tilde{f}(x, y, s, t) \geq f(x, y, s, t) \geq 0$.

Theorem 2.1. If inequality (1.3) holds for the nonnegative function $u(x, y)$, then

$$
u(x, y) \leq \psi^{-1}\left\{W_{2}^{-1}[\Xi(x, y)]\right\}
$$

for all $(x, y) \in\left[x_{0}, X_{1}\right) \times\left[y_{0}, Y_{1}\right)$, where

$$
\begin{aligned}
& \Xi(x, y):=W_{2}\left[W_{1}^{-1}\left(r_{2}(x, y)\right)\right]+\int_{b\left(x_{0}\right)}^{b(x)} \int_{c\left(y_{0}\right)}^{c(y)} \tilde{f}(x, y, s, t)\left[\int_{b\left(x_{0}\right)}^{s} \int_{c\left(y_{0}\right)}^{t} g(s, t, \tau, \sigma) d \tau d \sigma\right] d t d s, \\
& r_{2}(x, y):=W_{1}\left(r_{1}(x, y)\right)+\int_{b\left(x_{0}\right)}^{b(x)} \int_{c\left(y_{0}\right)}^{c(y)} \tilde{f}(x, y, s, t) d t d s, \\
& r_{1}(x, y):=a\left(x_{0}, y\right)+\int_{x_{0}}^{x}\left|a_{x}(s, y)\right| d s,
\end{aligned}
$$

and $\left(X_{1}, Y_{1}\right) \in \Lambda$ is arbitrarily given on the boundary of the planar region

$$
\mathcal{R}:=\left\{(x, y) \in \Lambda: \Xi(x, y) \in \operatorname{Dom}\left(W_{2}^{-1}\right), r_{2}(x, y) \in \operatorname{Dom}\left(W_{1}^{-1}\right)\right\}
$$

Here Dom denotes the domain of a function.

Proof. By the definition of functions $w_{i}$ and $\tilde{f}_{i}$, from (1.3) we get

$$
\begin{aligned}
& \psi(u(x, y)) \\
& \quad \leq a(x, y)+\int_{b\left(x_{0}\right)}^{b(x)} \int_{c\left(y_{0}\right)}^{c(y)} \tilde{f}(x, y, s, t)\left[w_{1}(u(s, t))+\int_{b\left(x_{0}\right)}^{s} \int_{c\left(y_{0}\right)}^{t} g(s, t, \sigma, \tau) w_{2}(u(\sigma, \tau)) d \tau d \sigma\right] d t d s
\end{aligned}
$$

for all $(x, y) \in \Lambda$.

Firstly, we discuss the case that $a(x, y)>0$ for all $(x, y) \in \Lambda$. It means that $r_{1}(x, y)>0$ for all $(x, y) \in \Lambda$. In such a circumstance, $r_{1}(x, y)$ is positive and nondecreasing on $\Lambda$ and

$$
r_{1}(x, y) \geq a\left(x_{0}, y\right)+\int_{x_{0}}^{x} a_{x}(t, y) d t
$$

Regarding (1.3), we consider the auxiliary inequality

$$
\begin{aligned}
& \psi(u(x, y)) \\
& \quad \leq r_{1}(x, y)+\int_{b\left(x_{0}\right)}^{b(x)} \int_{c\left(y_{0}\right)}^{c(y)} \tilde{f}(X, y, s, t)\left[w_{1}(u(s, t))+\int_{b\left(x_{0}\right)}^{s} \int_{c\left(y_{0}\right)}^{t} g(s, t, \sigma, \tau) w_{2}(u(\sigma, \tau)) d \tau d \sigma\right] d t d s
\end{aligned}
$$


for all $(x, y) \in\left[x_{0}, X\right) \times J$, where $x_{0} \leq X \leq X_{1}$ is chosen arbitrarily. We claim that

$$
\begin{aligned}
u(x, y) \leq \psi^{-1}\left\{W_{2}^{-1}[\right. & W_{2}\left(W_{1}^{-1}\left(W_{1}\left(r_{1}(x, y)\right)+\int_{b\left(x_{0}\right)}^{b(x)} \int_{c\left(y_{0}\right)}^{c(y)} \tilde{f}(X, y, s, t) d t d s\right)\right) \\
& \left.\left.+\int_{b\left(x_{0}\right)}^{b(x)} \int_{c\left(y_{0}\right)}^{c(y)} \tilde{f}_{1}(X, y, s, t)\left[\int_{b\left(x_{0}\right)}^{s} \int_{c\left(y_{0}\right)}^{t} g(s, t, \tau, \sigma) d \tau d \sigma\right] d t d s\right]\right\}
\end{aligned}
$$

for all $(x, y) \in\left[x_{0}, X\right) \times\left[y_{0}, Y_{1}\right)$, where $Y_{1}$ is defined by $(2.8)$.

Let $\eta(x, y)$ denote the right-hand side of (2.11), which is a nonnegative and nondecreasing function on $\left[x_{0}, X\right) \times J$. Then, $(2.11)$ is equivalent to

$$
u(x, y) \leq \psi^{-1}(\eta(x, y)) \quad \forall(x, y) \in\left[x_{0}, Y\right) \times J
$$

By the fact that $b(x) \leq x$ for $x \in\left[x_{0}, X\right)$ and the monotonicity of $w_{i}, \psi, \eta$, and $b(x)$, we have

$$
\begin{aligned}
& \frac{(\partial / \partial x) \eta(x, y)}{w_{1}\left(\psi^{-1}(\eta(x, y))\right)} \\
& \leq \frac{(\partial / \partial x) r_{1}(x, y)}{w_{1}\left(\psi^{-1}\left(r_{1}(x, y)\right)\right)}+\frac{b^{\prime}(x)}{w_{1}\left(\psi^{-1}(\eta(x, y))\right)} \\
& \quad \times \int_{c\left(y_{0}\right)}^{c(y)} \tilde{f}_{1}(X, y, b(x), t)\left[w_{1}(u(b(x), t))+\int_{b\left(x_{0}\right)}^{b(x)} \int_{c\left(y_{0}\right)}^{t} g(b(x), t, \tau, \sigma) w_{2}(u(\tau, \sigma)) d \tau d \sigma\right] d t \\
& \leq \frac{(\partial / \partial x) r_{1}(x, y)}{w_{1}\left(\psi^{-1}\left(r_{1}(x, y)\right)\right)}+b^{\prime}(x) \int_{c\left(y_{0}\right)}^{c(y)} \tilde{f}_{1}(X, y, b(x), t) d t \\
& \quad+b^{\prime}(x) \int_{c\left(y_{0}\right)}^{c(y)} \tilde{f}_{1}(X, y, b(x), t)\left[\int_{b\left(x_{0}\right)}^{b(x)} \int_{c\left(y_{0}\right)}^{t} g(b(x), t, \tau, \sigma) \phi(u(\tau, \sigma)) d \tau d \sigma\right] d t
\end{aligned}
$$

for all $(x, y) \in\left[x_{0}, X\right) \times J$. Integrating the above from $x_{0}$ to $x$, we get

$$
\begin{aligned}
W_{1}(\eta(x, y)) \leq & W_{1}\left(r_{1}(x, y)\right)+\int_{b\left(x_{0}\right)}^{b(x)} \int_{c\left(y_{0}\right)}^{c(y)} \tilde{f}_{1}(X, y, s, t) d t d s \\
& +\int_{b\left(x_{0}\right)}^{b(x)} \int_{c\left(y_{0}\right)}^{c(y)} \tilde{f}_{1}(X, y, s, t)\left[\int_{b\left(x_{0}\right)}^{s} \int_{c\left(y_{0}\right)}^{t} g(s, t, \tau, \sigma) \phi(u(\tau, \sigma)) d \tau d \sigma\right] d t d s
\end{aligned}
$$

for all $(x, y) \in\left[x_{0}, X\right) \times J$. Let

$$
\begin{gathered}
\psi(\xi(x, y)):=W_{1}(\eta(x, y)) \\
\tilde{r}_{2}(x, y):=W_{1}\left(r_{1}(x, y)\right)+\int_{b\left(x_{0}\right)}^{b(x)} \int_{c\left(y_{0}\right)}^{c(y)} \tilde{f}_{1}(X, y, s, t) d t d s
\end{gathered}
$$


From (2.15), (2.16), we obtain

$$
\begin{aligned}
& \psi(\xi(x, y)) \\
& \quad \leq \tilde{r}_{2}(x, y)+\int_{b\left(x_{0}\right)}^{b(x)} \int_{c\left(y_{0}\right)}^{c(y)} \tilde{f}_{1}(X, y, s, t)\left[\int_{b\left(x_{0}\right)}^{s} \int_{c\left(y_{0}\right)}^{t} g(s, t, \tau, \sigma) \phi(u(\tau, \sigma)) d \tau d \sigma\right] d t d s
\end{aligned}
$$

for all $x_{0} \leq x<X, y_{0} \leq y<y_{1}$. Let $\beta(x, y)$ denote the right-hand side of (2.17), which is a nonnegative and nondecreasing function on $\left[x_{0}, Y\right) \times J$. Then, $(2.17)$ is equivalent to

$$
\psi(\xi(x, y)) \leq \beta(x, y) \quad \forall(x, y) \in\left[x_{0}, Y\right) \times J
$$

From (2.13), (2.16), and (2.18), we have

$$
u(x, y) \leq \psi^{-1}(\eta(x, y))=\psi^{-1}\left(W_{1}^{-1}(\psi(\xi(x, y))) \leq \psi^{-1}\left(W_{1}^{-1}(\beta(x, y))\right)\right.
$$

for all $x_{0} \leq x<X, y_{0} \leq y<Y_{1}$, where $Y_{1}$ is defined by (2.8). By the definitions of $\phi, \psi$, and $W_{1}$, $\phi\left(\psi^{-1}\left(W_{1}^{-1}(s)\right)\right)$ is continuous and nondecreasing on $[0, \infty)$ and satisfies $\phi\left(\psi^{-1}\left(W_{1}^{-1}(s)\right)\right)>0$ for $s>0$. Let $h(s)=\psi^{-1}\left(W_{1}^{-1}(s)\right)$. Since $b^{\prime}(x) \geq 0$ and $b(x) \leq x$ for $x \in\left[x_{0}, X\right)$, from (2.19) we have

$$
\begin{aligned}
& \frac{(\partial / \partial x) \beta(x, y)}{\phi(h(\beta(x, y)))} \\
& \quad \leq \frac{(\partial / \partial x) \tilde{r}_{2}(x, y)}{\phi\left(h\left(\tilde{r}_{2}(x, y)\right)\right)} \\
& \quad+\frac{b^{\prime}(x)}{\phi(h(\beta(x, y)))} \int_{c\left(y_{0}\right)}^{c(y)} \tilde{f}_{1}(X, y, b(x), t)\left[\int_{b\left(x_{0}\right)}^{b(x)} \int_{c\left(y_{0}\right)}^{t} g(b(x), t, \tau, \sigma) \phi(u(\tau, \sigma)) d \tau d \sigma\right] d t d s \\
& \quad \leq \frac{(\partial / \partial x) \tilde{r}_{2}(x, y)}{\phi\left(h\left(\tilde{r}_{2}(x, y)\right)\right)}+b^{\prime}(x) \int_{c\left(y_{0}\right)}^{c(y)} \tilde{f}_{1}(X, y, b(x), t)\left[\int_{b\left(x_{0}\right)}^{b(x)} \int_{c\left(y_{0}\right)}^{t} g(b(x), t, \tau, \sigma) d \tau d \sigma\right] d t d s
\end{aligned}
$$

for all $(x, y) \in\left[x_{0}, X\right) \times\left[y_{0}, Y_{1}\right)$. Integrating the above from $x_{0}$ to $x$, by (2.4) we get

$$
\Phi(\beta(x, y)) \leq \Phi\left(\tilde{r}_{2}(x, y)\right)+\int_{b\left(x_{0}\right)}^{b(x)} \int_{c\left(y_{0}\right)}^{c(y)} \tilde{f}_{1}(X, y, s, t)\left[\int_{b\left(x_{0}\right)}^{s} \int_{c\left(y_{0}\right)}^{t} g(s, t, \tau, \sigma) d \tau d \sigma\right] d t d s
$$

for all $(x, y) \in\left[x_{0}, X\right) \times\left[y_{0}, y_{1}\right)$. By (2.19) and the above inequality, we obtain

$$
\begin{aligned}
& u(x, y) \\
& \leq \psi^{-1}\left\{W_{1}^{-1}\left[\Phi^{-1}\left(\Phi\left(\tilde{r}_{2}(x, y)\right)+\int_{b\left(x_{0}\right)}^{b(x)} \int_{c\left(y_{0}\right)}^{c(y)} \tilde{f}_{1}(X, y, s, t)\left[\int_{b\left(x_{0}\right)}^{s} \int_{c\left(y_{0}\right)}^{t} g(s, t, \tau, \sigma) d \tau d \sigma\right] d t d s\right)\right]\right\}
\end{aligned}
$$


for all $(x, y) \in\left[x_{0}, X\right) \times\left[y_{0}, Y_{1}\right)$, where $Y_{1}$ is defined by (2.8). It follows from (2.5) that

$$
\begin{aligned}
u(x, y) \leq \psi^{-1}\left\{W_{2}^{-1}[\right. & W_{2}\left(W_{1}^{-1}\left(W_{1}\left(r_{1}(x, y)\right)+\int_{b\left(x_{0}\right)}^{b(x)} \int_{c\left(y_{0}\right)}^{c(y)} \tilde{f}_{1}(X, y, s, t) d t d s\right)\right) \\
& \left.\left.+\int_{b\left(x_{0}\right)}^{b(x)} \int_{c\left(y_{0}\right)}^{c(y)} \tilde{f}_{1}(X, y, s, t)\left[\int_{b\left(x_{0}\right)}^{s} \int_{c\left(y_{0}\right)}^{t} g(s, t, \tau, \sigma) d \tau d \sigma\right] d t d s\right]\right\},
\end{aligned}
$$

which proves the claimed (2.12).

We start from the original inequality (1.3) and see that

$$
\begin{aligned}
& \psi(u(X, y)) \\
& \quad \leq r_{1}(X, y)+\int_{b\left(x_{0}\right)}^{b(X)} \int_{c\left(y_{0}\right)}^{c(y)} \tilde{f}(X, y, s, t)\left[\varphi_{1}(u(s, t))+\int_{b\left(x_{0}\right)}^{s} \int_{c\left(y_{0}\right)}^{t} g(s, t, \sigma, \tau) \varphi_{2}(u(\sigma, \tau)) d \tau d \sigma\right] d t d s
\end{aligned}
$$

for all $y \in\left[y_{0}, Y_{1}\right)$; namely, the auxiliary inequality (2.11) holds for $x=X, y \in\left[y_{0}, Y_{1}\right)$. By (2.12), we get

$$
\begin{aligned}
u(X, y) \leq \psi^{-1}\left\{W_{2}^{-1}[\right. & W_{2}\left(W_{1}^{-1}\left(W_{1}\left(r_{1}(X, y)\right)+\int_{b\left(x_{0}\right)}^{b(X)} \int_{c\left(y_{0}\right)}^{c(y)} \tilde{f}_{1}(X, y, s, t) d t d s\right)\right) \\
& \left.\left.+\int_{b\left(x_{0}\right)}^{b(X)} \int_{c\left(y_{0}\right)}^{c(y)} \tilde{f}_{1}(X, y, s, t)\left[\int_{b\left(x_{0}\right)}^{s} \int_{c\left(y_{0}\right)}^{t} g(s, t, \tau, \sigma) d \tau d \sigma\right] d t d s\right]\right\}
\end{aligned}
$$

for all $x_{0} \leq X \leq X_{1}, y_{0} \leq y \leq Y_{1}$. This proves (2.6).

The remainder case is that $a(x, y)=0$ for some $(x, y) \in \Lambda$. Let

$$
r_{1, \varepsilon}(x, y):=r_{1}(x, y)+\varepsilon,
$$

where $\varepsilon>0$ is an arbitrary small number. Obviously, $r_{1, \varepsilon}(x, y)>0$ for all $(x, y) \in \Lambda$. Using the same arguments as above, where $r_{1}(x, y)$ is replaced with $r_{1, \varepsilon}(x, y)$, we get

$$
\begin{aligned}
u(x, y) \leq \psi^{-1}\left\{W_{2}^{-1}[\right. & W_{2}\left(W_{1}^{-1}\left(W_{1}\left(r_{1, \varepsilon}(x, y)\right)+\int_{b\left(x_{0}\right)}^{b(x)} \int_{c\left(y_{0}\right)}^{c(y)} \tilde{f}_{1}(x, y, s, t) d t d s\right)\right) \\
& \left.\left.+\int_{b\left(x_{0}\right)}^{b(x)} \int_{c\left(y_{0}\right)}^{c(y)} \tilde{f}_{1}(x, y, s, t)\left[\int_{b\left(x_{0}\right)}^{s} \int_{c\left(y_{0}\right)}^{t} g(s, t, \tau, \sigma) d \tau d \sigma\right] d t d s\right]\right\}
\end{aligned}
$$

for all $x_{0} \leq X \leq X_{1}, y_{0} \leq y \leq Y_{1}$. Letting $\varepsilon \rightarrow 0_{+}$, we obtain (2.6) because of continuity of $r_{1, \varepsilon}$ in $\varepsilon$ and continuity of $\psi^{-1}, W_{1}^{-1}, W, W_{2}^{-1}$, and $W_{2}$. This completes the proof. 


\section{Applications}

In [11], the partial integrodifferential equation (1.2) was discussed for boundedness and uniqueness of the solutions under the assumptions that

$$
\begin{gathered}
|F(x, y, u, v)| \leq f(x, y)[|u|+|v|] \\
|h(x, y, s, t, u(s, t))| \leq g(x, y, s, t)|u(s, t)| \\
\left|F\left(x, y, u_{1}, v_{1}\right)-F\left(x, y, u_{2}, v_{2}\right)\right| \leq f(x, y)\left[\left|u_{1}-u_{2}\right|+\left|v_{1}-v_{2}\right|\right] \\
\left|h\left(x, y, s, t, u_{1}\right)-h\left(x, y, s, t, u_{2}\right)\right| \leq g(x, y, s, t)\left|u_{1}-u_{2}\right|
\end{gathered}
$$

respectively. In this section, we further consider the nonlinear delay partial integrodifferential equation

$$
\begin{gathered}
u_{x y}(x, y)=F\left(x, y, u(b(x), c(y)), \int_{b\left(b\left(x_{0}\right)\right)}^{b(x)} \int_{c\left(c\left(y_{0}\right)\right)}^{c(y)} h(b(x), c(y), \tau, \sigma, u(\tau, \sigma)) d \tau d \sigma\right), \\
u\left(x, y_{0}\right)=\alpha(x), \quad u\left(x_{0}, y\right)=\beta(y)
\end{gathered}
$$

for all $(x, y) \in \Lambda$, where $b, c$, and $u$ are supposed to be as in Theorem $2.1 ; h: \Lambda^{2} \times \mathbf{R} \rightarrow \mathbf{R}$, $F: \Lambda \times \mathbf{R}^{2} \rightarrow \mathbf{R}, \alpha: I \rightarrow \mathbf{R}$, and $\beta: J \rightarrow \mathbf{R}$ are all continuous functions such that $\alpha(0)=\beta(0)=0$. Obviously, the estimation obtained in [11] cannot be applied to (3.2).

We first give an estimation for solutions of (3.2) under the condition

$$
\begin{gathered}
|F(x, y, u, v)| \leq f(x, y)\left[\varphi_{1}(|u|)+|v|\right] \\
|h(x, y, s, t, u(s, t))| \leq g(x, y, s, t)\left|\varphi_{2}(u(s, t))\right| .
\end{gathered}
$$

Corollary 3.1. If $|\alpha(x)+\beta(y)|$ is nondecreasing in $x$ and $y$ and (3.3) holds, then every solution $u(m, n)$ of (3.2) satisfies

$$
u(x, y) \leq W_{2}^{-1}[\Xi(x, y)] \quad \forall(x, y) \in\left[x_{0}, X_{1}\right) \times\left[y_{0}, Y_{1}\right)
$$

where

$$
\begin{aligned}
\Xi(x, y):= & W_{2}\left\{W_{1}^{-1}\left[W_{1}(|\alpha(x)+\beta(y)|)+\int_{b\left(x_{0}\right)}^{b(x)} \int_{c\left(y_{0}\right)}^{c(y)} \frac{f\left(b^{-1}(s), c^{-1}(t)\right)}{b^{\prime}\left(b^{-1}(s)\right) c^{\prime}\left(c^{-1}(t)\right)} d t d s\right]\right\} \\
& +\int_{b\left(x_{0}\right)}^{b(x)} \int_{c\left(y_{0}\right)}^{c(y)} \frac{f\left(b^{-1}(s), c^{-1}(t)\right)}{b^{\prime}\left(b^{-1}(s)\right) c^{\prime}\left(c^{-1}(t)\right)}\left[\int_{b\left(x_{0}\right)}^{s} \int_{c\left(y_{0}\right)}^{t} g(s, t, \tau, \sigma) d \tau d \sigma\right] d t d s,
\end{aligned}
$$

and $W_{1}, W_{1}^{-1}, W_{2}, W_{2}^{-1}$, and $X_{1}, Y_{1}$ are defined as in Theorem 2.1 
Corollary 3.1 actually gives a condition of boundedness for solutions. Concretely, if there is a positive constant $M$ such that

$$
\begin{aligned}
& |\alpha(x)+\beta(y)|<M, \quad \int_{b\left(x_{0}\right)}^{b(x)} \int_{c\left(y_{0}\right)}^{c(y)} \frac{f\left(b^{-1}(s), c^{-1}(t)\right)}{b^{\prime}\left(b^{-1}(s)\right) c^{\prime}\left(c^{-1}(t)\right)} d t d s<M, \\
& \int_{b\left(x_{0}\right)}^{b(x)} \int_{c\left(y_{0}\right)}^{c(y)} \frac{f\left(b^{-1}(s), c^{-1}(t)\right)}{b^{\prime}\left(b^{-1}(s)\right) c^{\prime}\left(c^{-1}(t)\right)}\left[\int_{b\left(x_{0}\right)}^{s} \int_{c\left(y_{0}\right)}^{t} g(s, t, \tau, \sigma) d \tau d \sigma\right] d t d s<M
\end{aligned}
$$

on $\left[x_{0}, X_{1}\right) \times\left[y_{0}, Y_{1}\right)$, then every solution $u(x, y)$ of $(3.2)$ is bounded on $\left[x_{0}, X_{1}\right) \times\left[y_{0}, Y_{1}\right)$.

Next, we give the condition of the uniqueness of solutions for (3.2).

Corollary 3.2. Suppose

$$
\begin{gathered}
\left|F\left(x, y, u_{1}, v_{1}\right)-F\left(x, y, u_{2}, v_{2}\right)\right| \leq f(x, y)\left[\varphi_{1}\left(\left|u_{1}-u_{2}\right|\right)+\left|v_{1}-v_{2}\right|\right] \\
\left|h\left(x, y, s, t, u_{1}\right)-h\left(x, y, s, t, u_{2}\right)\right| \leq g(x, y, s, t) \varphi_{2}\left(\left|u_{1}-u_{2}\right|\right)
\end{gathered}
$$

where $f, g, \varphi_{1}, \varphi_{2}$ are defined as in Theorem 2.1. There is a positive number $M$ such that

$$
\begin{aligned}
& \int_{b\left(x_{0}\right)}^{b(x)} \int_{c\left(y_{0}\right)}^{c(y)} \frac{f\left(b^{-1}(s), c^{-1}(t)\right)}{b^{\prime}\left(b^{-1}(s)\right) c^{\prime}\left(c^{-1}(t)\right)} d t d s<M, \\
& \int_{b\left(x_{0}\right)}^{b(x)} \int_{c\left(y_{0}\right)}^{c(y)} \frac{f\left(b^{-1}(s), c^{-1}(t)\right)}{b^{\prime}\left(b^{-1}(s)\right) c^{\prime}\left(c^{-1}(t)\right)}\left[\int_{b\left(x_{0}\right)}^{s} \int_{c\left(y_{0}\right)}^{t} g(s, t, \tau, \sigma) d \tau d \sigma\right] d t d s<M
\end{aligned}
$$

on $\left[x_{0}, X_{1}\right) \times\left[y_{0}, Y_{1}\right)$. Then, (3.2) has at most one solution on $\left[x_{0}, X_{1}\right) \times\left[y_{0}, Y_{1}\right)$, where $X_{1}, Y_{1}$ are defined as in Theorem 2.1 .

\section{Acknowledgments}

This work is supported by the Scientific Research Fund of Guangxi Provincial Education Department (no. 200707MS112), the Natural Science Foundation (no. 2006N001), and the Applied Mathematics Key Discipline Foundation of Hechi College of China.

\section{References}

[1] R. Bellman, "The stability of solutions of linear differential equations," Duke Mathematical Journal, vol. 10, no. 4, pp. 643-647, 1943.

[2] T. H. Gronwall, "Note on the derivatives with respect to a parameter of the solutions of a system of differential equations," The Annals of Mathematics, vol. 20, no. 4, pp. 292-296, 1919.

[3] R. P. Agarwal, S. Deng, and W. Zhang, "Generalization of a retarded Gronwall-like inequality and its applications," Applied Mathematics and Computation, vol. 165, no. 3, pp. 599-612, 2005.

[4] I. Bihari, "A generalization of a lemma of Bellman and its application to uniqueness problems of differential equations," Acta Mathematica Hungarica, vol. 7, pp. 81-94, 1956.

[5] W.-S. Cheung, "Some new nonlinear inequalities and applications to boundary value problems," Nonlinear Analysis: Theory, Methods E Applications, vol. 64, no. 9, pp. 2112-2128, 2006.

[6] C. M. Dafermos, "The second law of thermodynamics and stability," Archive for Rational Mechanics and Analysis, vol. 70, no. 2, pp. 167-179, 1979. 
[7] F. M. Dannan, "Integral inequalities of Gronwall-Bellman-Bihari type and asymptotic behavior of certain second order nonlinear differential equations," Journal of Mathematical Analysis and Applications, vol. 108, no. 1, pp. 151-164, 1985.

[8] R. Medina and M. Pinto, "On the asymptotic behavior of solutions of certain second order nonlinear differential equations," Journal of Mathematical Analysis and Applications, vol. 135, no. 2, pp. 399-405, 1988.

[9] D. S. Mitrinović, J. E. Pečarić, and A. M. Fink, Inequalities Involving Functions and Their Integrals and Derivatives, vol. 53 of Mathematics and Its Applications, Kluwer Academic Publishers, Dordrecht, The Netherlands, 1991.

[10] B. G. Pachpatte, Inequalities for Differential and Integral Equations, vol. 197 of Mathematics in Science and Engineering, Academic Press, San Diego, Calif, USA, 1998.

[11] B. G. Pachpatte, "Bounds on certain integral inequalities," Journal of Inequalities in Pure and Applied Mathematics, vol. 3, no. 3, article 47, 10 pages, 2002.

[12] W.-S. Wang, "A generalized sum-difference inequality and applications to partial difference equations," Advances in Difference Equations, vol. 2008, Article ID 695495, 12 pages, 2008. 